\title{
Development of Checkpoint Inhibitors for Sarcomas
}

\author{
Rodrigo R Munhoz, ${ }^{1,2}$ William D Tap, ${ }^{3,4}$ and Sandra P D'Angelo ${ }^{3,4}$ \\ 1. Oncology Center, Hospital Sírio Libanês, São Paulo, Brazil; 2. Instituto do Cancer do Estado de São Paulo, Universidade de São Paulo, São Paulo, Brazil; \\ 3. Department of Medicine, Memorial Sloan Kettering Cancer Center, New York, US; 4. Weill Cornell Medical College, New York, US
}

DOI: https://doi.org/10.17925/OHR.2017.13.01.25

S arcomas comprise a rare and heterogeneous group of malignancies of bone and soft tissue origin. Despite optimal approach, a significant proportion of patients will develop recurrent/metastatic disease. Although advances have been achieved, therapeutic options for these patients are limited and prognosis remains poor. Over the past century, the characterization of mechanisms involved in the interaction between tumor cells and the immune system has paved the way for the development of different forms of cancer immunotherapy, including cytokines, vaccines, cell therapies, and, more recently and successfully, monoclonal antibodies against molecules involved in the modulation of immune response, or immune checkpoint inhibitors. While the clinical applicability of this approach has been limited in sarcomas, the immunogenic potential of this group of malignancies was demonstrated more than 100 years ago. In this article, we review aspects associated with the immunogenicity of sarcomas and how the use of checkpoint inhibitors is being explored for this group of patients.

\section{Keywords}

Soft tissue sarcomas, immunotherapy, anti-PD-1, immune checkpoint blockade

Disclosure: Rodrigo R Munhoz, William D Tap, and Sandra P D'Angelo have nothing to disclose in relation to this article. No funding was received for the publication of this article. This study involves a review of the literature and did not involve any studies with human or animal subjects performed by any of the authors.

Authorship: All named authors meet the International Committee of Medical Journal Editors (ICMJE) criteria for authorship of this manuscript, take responsibility for the integrity of the work as a whole, and have given final approval to the version to be published.

open Access: This article is published under the Creative Commons Attribution Noncommercial License, which permits any non-commercial use, distribution, adaptation and reproduction provided the original author(s) and source are given appropriate credit.

Received: March 1, 2017

Accepted: April 12, 2017

Citation: Oncology \& Hematology Review, 2017:13(1):25-9

Corresponding Author: Sandra P D'Angleo, Sarcoma Medical Oncology Service, 300 East 66th Street, New York, NY 10065, US. E: dangelos@mskcc.org
Sarcomas consist of a highly diverse group of malignancies of mesenchymal origin, encompassing more than 80 distinct subtypes and diagnosed in approximately 15,000 patients every year in the US.

Surgery still represents the mainstay of therapy for patients with localized disease. Although the use of multimodal treatment with curative intent remains debatable in soft tissue sarcomas (STS), radiation therapy and chemotherapy are often used in the neo- or adjuvant settings in select situations; for bone sarcomas, combination regimens in association with surgical resection remain the standard treatment. ${ }^{2}$ For patients with advanced (unresectable or metastatic) disease, however, the prognosis remains poor, and median survival rarely exceeds 12-15 months. ${ }^{2}$ Alternatives for patients with sarcomas not amenable to treatment with curative intent still rely on cytotoxic agents; standard chemotherapy such as doxorubicin, ifosfamide, and dacarbazine result in objective responses in 10-30\% of the patients, usually of short duration, and the efficacy of these agents is largely influenced by the histologic subtype and tumor grade. ${ }^{3.4}$ During the past years, new options became available for clinical use, including pazopanib, trabectedin, eribulin, and olaratumab. Of note, olaratumab, a monoclonal antibody against the subunit alpha of the plateletderived growth factor receptor (PDGFR), resulted in an 11.8 month-overall survival improvement (hazard ratio [HR] 0.46; 95\% confidence interval [Cl] 0.30-0.71; $\mathrm{p}=0.0003$ ) when used in combination with doxorubicin, versus doxorubicin alone, in a randomized, phase II trial. ${ }^{5}$ Although survival gains have indeed been achieved, ${ }^{5,6}$ improvements have been modest and short lived in most scenarios and the therapeutic development has been slower in comparison to other solid tumors, highlighting the need for new, effective treatment options for this group of patients.

Manipulation of the immune system has emerged as a new hallmark of cancer therapeutics during the past decade, although observations of tumor regressions mediated by what is now characterized as an anticancer immune response date back to the nineteenth century. ${ }^{7.8}$ Different approaches to harness the immune system have been investigated: while cytokines, vaccines, and adoptive cell therapy with artificially engineered antigen receptors (or chimeric antigen receptor [CAR] T cells) or modified T cell receptors (TCRS) resulted in variable degrees of antitumor effect, the most practicechanging and clinically applicable development in the management of solid tumors resulted from the use of monoclonal antibodies targeting molecules involved in the modulation of immune activation and response, or checkpoints. 9.10 
In physiologic conditions, antigens or peptides derived from potential pathogens (bacteria, viruses, tumor cells, etc.) are processed and expressed by antigen-presenting cells (dendritic cells, macrophages, and B cells) through the major histocompatibility complex that engages T cells through the TCR, eliciting a complex sequence of events that culminate with the activation of both innate and adaptive immunity. ${ }^{10,11}$ Key players of cell-mediated adaptive responses are $\mathrm{T}$ cells with either helper cells (CD4+ T cells) or cytotoxic capabilities (CD8+ T cells), as well as memory cells involved in sustained immunity.12 Across different steps of this cycle, the magnitude, duration, and, ultimately, efficacy, of the immune response are influenced by modulatory mechanisms, which can result in either amplification or abrogation of this cycle. While essential in avoiding uncontrolled immune responses and autoimmunity in physiologic situations, these negative regulatory pathways can be exploited by tumor cells as immune evasion mechanisms. ${ }^{13,14}$ As examples of cell surface molecules involved in immunosuppressive singling pathways, cytotoxic T-lymphocyte associated protein-4 (CTLA-4, or CD152) and programmed cell death receptor-1 (PD-1, or CD274) or its ligand (PD-L1) have been successfully targeted by inhibitory monoclonal antibodies, or immune checkpoint inhibitors (ICIs), resulting in a paradigm shift in the management of a growing number of malignancies. ${ }^{15,16}$ The ICl ipilimumab, a human monoclonal antibody that binds to CTLA-4; pembrolizumab and nivolumab, molecules that target PD-1; and atezoluzimab, an anti-PD-L1 agent, have all been approved for clinical use. In addition to response rates varying from $10 \%$ to more than $50 \%$, ICIs have been shown to allow for sustained disease control and long-lasting immune-mediated responses, ${ }^{15-21}$ and striking activity resulted from combined CTLA-4 and PD-1 blockade.22,23

Interestingly enough, preliminary observations of tumor regressions following wound infections and erysipelas by Busch and Coley alluded to patients with sarcomas. ${ }^{7.8}$ Antitumor activity has been further demonstrated in sarcomas with the use of various forms of immunotherapy. ${ }^{24}$ As examples, cytokine therapy with interleukin-2 (II-2) and interferons lead to occasional responses in patients with heavily pretreated Ewing sarcoma (ES) and osteosarcoma. ${ }^{25-29}$ Similarly, liposomal muramyl tripeptide phosphatidylethanolamine (L-MTP-PE), a muramyl dipeptide analogue associated with enhanced NK-к $\beta$ signaling and monocyte/macrophage activation, is approved for clinical use in Europe (although not by the US Food and Drug Administration) based on survival improvements in a randomized trial investigating the addition of this agent to conventional chemotherapy in patients with osteosarcoma receiving adjuvant treatment..$^{30}$ Trabectedin, a compound with a complex mechanism of action currently approved for the treatment of sarcomas, was shown to affect macrophage viability, differentiation, and the production of CCL2 and IL-6, suggesting an immune-mediated effect that could potentially be involved in antitumor activity. ${ }^{31}$ Additional proofs of the concept that mobilization of the immune system can result in antitumor effect in sarcoma patients are plentiful in the literature, deriving from different forms of vaccines, tumor antigens/peptides, lysates, and, more recently, TCR-transduced T cells specific to NY-ESO-1 in synovial sarcomas. ${ }^{32-34}$

Evidence addressing the efficacy of monoclonal antibodies against immune checkpoints begins to emerge, as recent studies have yielded both promising and disappointing results across different histologies. In this review, we revisit the mechanisms underlying the immunogenic potential of sarcomas, the currently available clinical data regarding the efficacy of ICls in sarcomas, as well as future directions.

\section{Is there a rationale for the use of checkpoint inhibition in sarcomas?}

\section{Preliminary evidence of a cancer-immune interaction in sarcomas}

Although the early experiments by coley were never consolidated as a feasible and applicable treatment option, the potential role of the immune system in sarcoma control and progression is well characterized. A higher incidence of sarcomas has been reported in immunodeficient patients, including those with severe combined immunodeficiency, Wiskott-Aldrich syndrome, ataxia-telangiectasia, and in the setting of HIV infection.35,36 Likewise, allograft transplant recipients exhibit a higher risk of developing Kaposi sarcoma, leiomyosarcoma, rhabdomyosarcoma, and so forth. ${ }^{37}$ Conversely, a high density of CD20+ tumor-infiltrating lymphocytes (TILS) in resected STS was shown to be an independent positive prognostic factor for disease-specific survival. ${ }^{38}$ In ES, survival correlated with tumor infiltrating $\mathrm{T}$ cells, including CD8+ T cells, and expression of genes associated with proinflammatory chemokines (CXCR3, CCR5, CXCL9, CXCL10, and CCL5). ${ }^{39}$ The presence of TILS and a correlation with prognosis has also been documented in a variety of sarcoma subtypes, including liposarcoma, chordomas, angiosarcomas, synovial sarcoma, and undifferentiated pleomorphic sarcoma (UPS). ${ }^{40-43}$

Another example of the potential activity of immune system against sarcomas results from observations of patients with gastrointestinal stromal tumors (GISTS) treated with imatinib. In addition to the wellcharacterized antitumor effect resulting from tyrosine kinase "on-target" KIT inhibition, benefit from imatinib therapy was associated with increased intratumoral CD8+ T cells, mobilization of natural killer (NK) cells from the stroma into the tumor, and depletion of cells with suppressive functions, through apoptosis of tumor-infiltrating regulatory $T$ cells (Tregs). ${ }^{44}$ In addition, imatinib has been shown to result in inhibition of indolamine 2,3-dioxygenase (IDO), an intracellular enzyme involved in tryptophan metabolism and immunosuppression upon lymphocyte activation. ${ }^{45}$

\section{Tumor antigens and intrinsic antigenic potential in sarcomas}

The tumor intrinsic immunogenic potential can be affected by a variety of factors, and the diversity of sarcoma poses an additional challenge to the understanding and clinical translation of these variables in the setting of the use of immunotherapy. Antigens are the initial step in the elicitation of an effective and specific immune response. Tumor antigens can be classified as tumor-specific antigens, that result from cancer-germline genes, point mutations, or oncogenic viruses and unique to tumor cells, or tumor-associated antigens (TAAS), which include differentiation antigens and peptides associated with genes overexpressed in tumors. ${ }^{46,47}$ Among the large family of TAAs, expression of cancer testis antigens (NY-ESO, MAGE-A3, MAGE-A4, PRAME, and LAGE) occurs in a variety of sarcomas, including synovial sarcomas, myxoid round-cell liposarcoma, osteosarcoma, ES, chondrosarcoma, leiomyosarcoma, and UPS. ${ }^{48-50}$

Nevertheless, and as expected for such a heterogeneous group of tumors, immunogenicity is not uniform among sarcomas, and can be influenced by the tumor genomic landscape or mutational load. Across different histologies, and particularly in melanoma and non-small cell-lung cancer, increased number of somatic missense mutations and mutational epitopes have been associated with patient survival and benefit from both anti-CTLA-4 and anti-PD-1 agents, ${ }^{19,51-54}$ although this remains a topic 
of ongoing research. While a subgroup of sarcomas (dedifferentiated liposarcomas, malignant peripheral nerve sheath tumor, angiosarcoma, etc.) is characterized by genome instability and a complex/unbalanced karyotype that results from nonspecific gains and losses and genomic aberrations, ${ }^{55,56}$ resulting in a potentially immunogenic phenotype, a large proportion of sarcomas (including ES, synovial sarcoma, etc.) exhibit simple karyotypic aberrations, including amplifications, deletions, or chromosomal translocations resulting in gene fusions, although genomic instability has been reported even in this setting. ${ }^{77}$ High levels of somatic copy number alterations (aneuploidy) have been shown to correlate with total number of mutations, elevated markers of cell cycle/proliferation, reduced expression of markers for cytotoxic immune cell infiltrates, and poorer prognosis in melanoma patients treated with anti-CTLA therapy, supporting the concept that aneuploidy is involved in mechanisms of cell proliferation and immune evasion..$^{58}$

\section{Immune infiltrates and PD-L1 expression in sarcomas}

In addition to its intrinsic immunogenic potential resulting from neoantigens and neoepitopes, several aspects involved in the tumor microenvironment and immune synapse may determine the magnitude and efficacy of a tumor-specific immune response. As previously cited, the characterization and distribution of the T cell infiltrate has been correlated with survival across different malignancies, including sarcomas, as well as potential benefit from anti-CLTA-4 and anti-PD-1 therapy. ${ }^{39,4,1,5,59-62}$ Other variables associated with innate and adaptive immune activation may serve as markers of treatment efficacy. Indeed, expression of PD-L1 by tumor cells or immune cells has been associated with increased response rates and prolonged survival in melanoma and nonsquamous non-small cell lung cancer, ${ }^{16,20,63}$ although the use of PD-L1 as a biomarker for clinical decisions remains debatable.

In sarcoma, expression of PD-L1 by tumor cells and immune cells has been described. In a mixed cohort of 105 sarcoma patients, PD-L1 expression occurred in $58 \%$ of STS tumor samples and, in combination with PD-1-positive lymphocyte intratumoral infiltration, correlated with advanced clinicopathologic parameters (advanced clinical stage and presence of metastatic disease, higher histologic grade, tumor necrosis, and poor differentiation) and worse overall survival and event-free survival by multivariate analysis. ${ }^{64}$ In another series that included 50 tumor samples including GIST $(n=14)$, liposarcoma $(n=5)$, leiomyosarcoma $(n=4)$, and other subtypes, PD-L1 expression $>1 \%$ was demonstrated in $12 \%$ of the cases, with the highest prevalence in GIST-29\%. In addition, PD-L1 expression by lymphocytes and macrophages in the tumor microenvironment occurred in $30 \%$ and $58 \%$, respectively. ${ }^{41}$ Nevertheless, no association between PD-L1 and prognosis was demonstrated. Using a multiplatform analysis of more than 2,000 sarcoma samples, Movva and colleagues demonstrated PD-L1 expression by immunohistochemistry in approximately $50 \%$ of the analyzed tumor samples, including leiomyosarcomas, liposarcomas, UPS, and chondrosarcomas. ${ }^{65}$ Using a similar approach to 65 sarcoma tumor samples, PD-L1 expression in tumor tissue and microenvironment was detected in $19(29 \%)$ and 20 cases (30\%), respectively. Although none of the five cases of ES of bone origin showed PD-1/PD-L1 expression, two of three cases of extraosseous ES were positive for PD-1 and PD-L1 expression. Positivity for PD-1 and/or PD-L1 in the tumor and/or microenvironment was also demonstrated in three of six cases with UPS, two of four cases with peripheral nerve sheath tumors, one of four cases with synovial sarcoma, and the majority (four or five) of Kaposi sarcoma samples. ${ }^{60}$ Additional markers of immune activation were evaluated by immunohistochemical staining in formalin-fixed, paraffin-embedded samples of 371 patients with localized primary STS treated with curative intent (UPS: 41\%; leiomyosarcoma: 34\%; myxofibrosarcoma: 61\%; dedifferentiated liposarcoma: 5\%). PD-L1 expression $\geq 1 \%$ occurred in 72 samples (19\%) and was mostly expressed by immune cells; while IDO expression by tumor cells $\geq 5 \%$ occurred in 152 (52\%) cases. Although the presence of PD-L1 and IDO was significantly associated with $\mathrm{CD} 8+\mathrm{T}$ cell infiltration, no correlation between expression of immune markers and prognosis was demonstrated. ${ }^{67}$ Nevertheless, it is important to highlight that using the expression of PD-L1 as a biomarker encases a series of challenges and unascertained aspects. While early clinical development of anti-PD-1 agents already suggested a correlation between PD-L1 expression and response to therapy, ${ }_{1}^{16}$ this correlation is imperfect. PD-L1 expression occurs along a spectrum of positivity and even tumors with low/negative expression of PD-L1 may respond to checkpoint inhibitors. ${ }^{63}$ In addition to analytical technical issues detecting PD-L1 (type of antibody used for immunohistochemistry staining, cut-off, components of the tumor microenvironment in which PD-L1 expression is measured, etc.), tumor heterogeneity also limits the interpretation of PD-L1 expression.

Taken together, these evidence support the immunogenic potential of sarcoma and provide the background for the investigation of ICI in this setting.

\section{Available clinical data of immune checkpoint inhibitors in sarcomas}

Despite the practice-changing results observed in patients with melanoma, non-small cell lung cancer, and other malignancies, less is known about the efficacy of $\mathrm{ICI}$ in sarcomas. While reports of activity of ipilimumab or anti-PD-1 agents against myxoid liposarcoma, ES, leiomyosarcoma, and so forth are available in the literature, few studies have examined ICI in a prospective manner and no randomized trials are available to date. ${ }^{6870}$

The anti-CTLA-4 agent ipilimumab, given at $3 \mathrm{mg} / \mathrm{kg}$ every 21 days for three doses, was evaluated in a small phase II study that included six pretreated patients with synovial sarcoma. ${ }^{71}$ The primary endpoint of the study was to determine the objective response rate (ORR) as per Response Evaluation Criteria In Solid Tumors (RECIST) criteria following three doses of ipilimumab; secondary endpoints were to determine the clinical benefit rate (objective responses + stable disease), evaluate NY-ESO-1 specific immunity and safety. There were no objective responses or evidence of significant immune reactivity to NY-ESO-1; median time to tumor progression was 1.85 months (range $0.47-2.1$ months) and the trial was terminated prematurely due to slow accrual and lack of activity. ${ }^{71}$ In a phase I, dose escalation trial of ipilimumab in pediatric patients with solid tumors, including 17 sarcoma patients, despite manageable toxicity, no objective responses were seen. Nevertheless, disease stabilization occurred in patients with osteosarcoma, clear cell sarcoma, and synovial sarcoma and an increased overall survival was seen in individuals with immune-related toxicities. ${ }^{72}$ Ipilimumab is also being investigated for patients with GIST in combination with tyrosine kinase inhibitors based on the immunologic "off-target" effects of imatinib and dasatinib previously described. In a recently published phase $\mathrm{Ib}$ trial that included 20 GIST patients and eight other sarcoma types, ipilimumab in combination with dasatinib was safely administered. Although no objective responses by RECIST or immunerelated response criteria (irRC) occurred, there were $7 / 13$ partial responses by Choi criteria. ${ }^{73}$ Ipilimumab is also being evaluated in combination with imatinib in an ongoing phase I trial (NCT01738139). 
Although still limited, data regarding the efficacy of PD-1 blockade in sarcomas start to emerge. Paoluzzi and colleagues reported the results of 28 patients ( 24 with STS and four with bone sarcomas) with advanced/ unresectable sarcomas treated with nivolumab $3 \mathrm{mg} / \mathrm{kg}$ every 2 weeks, through a patient assistance program from the manufacturer. ${ }^{74}$ Eighteen patients received concurrent pazopanib at 400-800 mg daily. Objective responses were seen in three cases: on dedifferentiated chondrosarcoma, one maxillary osteosarcoma, and one epithelioid sarcoma-of note, five patients developed grade 3-4 adverse events (colitis, pneumonitis, and elevation of aspartate transaminase [AST] and/or alanine transaminase $[\mathrm{ALT}])^{74}$ The largest experience with PD-1 blockade in sarcomas, however, comes from the SARC-028, a prospective, nonrandomized, phase II trial, recently presented following a preliminary analysis. Pembrolizumab was administered at $200 \mathrm{mg}$ given intravenously every 3 weeks to two concurrent arms: 40 patients were accrued in the STS cohort, with histologies limited to leiomyosarcoma $(n=10)$, dedifferentiated liposarcoma $(n=10)$, UPS $(n=10)$, and synovial sarcoma $(n=10)$, and 40 additional patients were included in the bone sarcoma arm (osteosarcoma, $n=21$; $E S, n=13$; dedifferentiated chondrosarcoma, $n=6$ ). The primary objective of the study was to determine the ORR as per RECIST criteria; secondary endpoints included safety/tolerability, overall survival, progression-free survival, correlation of pre-treatment PD-L1 expression with response, ORR by irRC and additional immunologic correlative studies. ${ }^{75}$ In the bone sarcoma arm, two objective responses were documented among 38 evaluable patients (ORR $=5 \%$; one patient with chondrosarcoma and one patient with osteosarcoma) and nine patients (24\%) achieved stable disease as best response. There were no objective responses among ES patients. In the interim analysis, three patients in the STS arm had not reached the first scan assessment. Among 37 evaluable STS patients, seven achieved objective responses (ORR=19\%), including 4/9 (44\%) patients with UPS, 2/9 (22\%) with dedifferentiated liposarcoma and $1 / 9$ (11\%) patients with synovial sarcoma; no objective responses occurred in the leiomyosarcoma arm. Stable disease was the best response in 15 patients (40\%), and 15 additional patients (40\%) developed progressive disease. Median PFS for the STS arm was 18 weeks, with an estimated 12-week PFS rate of $55 \% .^{75}$

Despite occasional reports of objective responses, ${ }^{70,76}$ the apparent lack of efficacy of PD-1 blockade in leiomyosarcomas was also suggested by another prospective study, in which nivolumab was administered at $3 \mathrm{mg} / \mathrm{kg}$ intravenously every 2 weeks to patients with metastatic leiomyosarcoma of uterine origin and at least one prior line of treatment. The trial was discontinued prematurely as no objective responses occurred among the first 12 enrolled patients after a median of five doses of nivolumab (range 2-6; median PFS 1.8 months). Of note, PD-L1 expression in tumor cells determined by immunohistochemistry was absent in the majority of tumor samples: among 10 archival tumor samples, one showed PD-L1 expression of $10 \%$ and one of $20 \%{ }^{77}$

\section{Future perspectives and conclusions}

In spite of the unequivocal markers of activation of both innate and adaptive immunity in sarcomas, so far the therapeutic manipulation of the immune system has not reproduced the same practice-changing results seen in other malignancies for this heterogeneous group of mesenchymal tumors. Understanding the mechanisms involved in primary resistance to immune checkpoint blockade would be instrumental in providing new frameworks for the development of immunotherapy in sarcomas-as an example, investigating the factors associated with the apparent lack of effectiveness of anti-PD-1 agents in leiomyosarcomas, which are marked by complex and potentially immunogenic molecular aberrations, would be of significant interest.

As results of studies investigating the efficacy of CTLA-4 and PD-1/ PD-L1 blockade in the management of sarcomas develop, with activity demonstrated in dedifferentiated liposarcoma and UPS, the role of distinct immunotherapeutic approaches must be considered in order to translate the immunogenic potential into clinical benefits. Monoclonal antibodies against distinct cell surface receptors with immunomodulatory properties with either co-stimulatory (CD137, OX40, CD27, GITR) or c0inhibitory (LAG-3, TIM-3, VISTA, BTLA) functions are being investigated, allowing for a growing number of combinations with potential synergistic activity.78,79 Approaches using combinations of checkpoint inhibitors are also being explored in sarcomas, and results of a phase II study of nivolumab alone or given concurrently with ipilimumab are awaited (NCT02500797). In addition, distinct forms of "immunotherapy" have shown antitumor effect in sarcoma patients. Adoptive cell therapies using genetically engineered lymphocytes resulted in promising and compelling activity in NY-ESO-1 expressing synovial sarcomas, and targeting specific epitopes overexpressed in sarcomas with modified antigen receptors fused with T cell intracellular signaling capabilities though CAR T cells may bring new alternatives. ${ }^{80,81}$ Similarly, advances in molecular biology and recombinant DNA technology allowed for the development of viruses with genetically altered, highly specific cytolytic properties. In addition to direct cytopathic effects caused by intracellular viral replication (oncolysis), genetic manipulation has allowed the development of strains capable of increasing cytotoxic activity and enhancing antitumor immune responses mediated by transcription products from engineered viral genome. Different strains of adenovirus, herpesvirus, vaccinia, and RNA viruses have been shown to be able to infect sarcoma cells and result in antitumor effect. ${ }^{82-84}$ Talimogene laherparepvec (T-VEC), an oncolytic herpesvirus delivered intralesionally, has been approved for clinical use in patients with melanoma, and the combination of T-VEC with $\mathrm{ICl}$ resulted in exciting results in early phase clinical trials. ${ }^{85,86}$ The role of combinations of immunotherapies with demethylating agents such as decitabine, capable of enhancing the expression of tumor antigens, is also being investigated. 87,88

Nevertheless, once again the biologic diversity of sarcomas will likely pose a major challenge for the development of the potential of immunotherapy in this setting. Moving forward will demand a better characterization of mechanisms involved in primary resistance and potential biomarkers, in order to identify subtypes of sarcomas more likely to respond to immunotherapy and to allow for a rational and successful design of clinical trials. $\square$ 
1. Siegel RL, Miller KD, Jemal A, Cancer statistics, 2015, CA Cancer J Clin, 2016;66:7-30

2. The ESMO/European Sarcoma Network Working Group, soft tissue and visceral sarcomas: ESMO clinical practice guidelines for diagnosis, treatment and follow up, Ann Oncol, 2014;25:102-12.

3. Van Glabbeke M, Van Oosterom AT, Oosterhuis JW, et al, Prognostic factors for the outcome of chemotherapy in advanced soft tissue sarcoma: an analysis of 2185 patients treated with anthracycline-containing first-line regimens - A European Organization for Research and Treatment of Cance Soft Tissue and Bone Sarcoma Group study, I Clin Oncol, 1999;17:150-7

4. Brennan MF, Antonescu CR, Maki RG, Management of Soft Tissue Sarcoma, New York: Springer, 2012.

5. Tap WD, Jones RL, Van Tine BA, et al., Olaratumab and doxorubicin versus doxorubicin alone for treatment of soft-tissue sarcoma: an open-label phase $1 \mathrm{~b}$ and randomised phase 2 trial, Lancet 2016;388:488-97.

6. Schöffski P, Chawla S, Maki RG, et al., Eribulin versus dacarbazine in previously treated patients with advanced liposarcoma or leiomyosarcoma: a randomised, open-label, multicentre, phase 3 trial, Lancet, 2016;387:1629-37.

7. Coley WB, II. Contribution to the knowledge of sarcoma, Ann surg, 1891;14:199-220

8. Curiel TJ, Historical Perspectives and Current Trends in Cancer Immunotherapy. In T.J. Curiel (ed), Cance Immunotherapy. Paradigms, Practice and Promise, New York: Springer, 2012

9. Rosenberg AS, Yang JC, Restifo NP, Cancer immunotherapy: moving beyond current vaccines, Nat Med, 2004:10:909-15.

10. Chen DS, Mellman I, Oncology meets immunology: the cancer-immunity cycle, Immunity, 2013:39:1-10.

11. Banchereau J, Steinman RM, Dendritic cells and the control of immunity, Nature, 1998;392:245-52.

12. Grakoui A, Bromley S, Sumen C, et al., The immunological synapse: a molecular machine controlling T cell activation, science, 1999;285:221-7.

13. Chen L, Flies DB, Molecular mechanisms of $\mathrm{T}$ cell co-stimulation and co-inhibition, Nat Rev Immunol, 2013:13:227-42.

14. Peggs KS, Quezada SA, Korman AJ, Allison JP, Principles and use of anti-CTLA4 antibody in human cancer immunotherapy, Curr Opin Immunol, 2006:18.206-13.

15. Hodi FS, O'Day SJ, MCDermott DF, et al., Improved survival with ipilimumab in patients with metastatic melanoma, N Eng/ J Med, 2010;363:711-23.

16. Topalian SL, Hodi FS, Brahmer JR, et al., Safety, activity and immune correlates of anti-PD1 antibody in cancer, N Eng/ J Med, 2012;366:2443-54.

17. Hodi FS, Kluger $\mathrm{H}$, Sznol M, et al., Durable, long-term survival in previously treated patients with advanced melanoma (MEL) who received nivolumab (NIVO) monotherapy in a phase I trial Presented at: AACR Annual Meeting, La Nouvelle Orleans Ballroom, Morial Convention Center, 17, April, 2016. Abstr no: СT001.

18. Ribas A, Hamid O, Daud A, et al., Association of pembrolizumab with tumor response and survival among patients with advanced melanoma, JAMA, 2016:315:1600-9.

19. Le ST, Uram JN, Wang H, et al., PD-1 blockade in tumors with mismatch repair deficiency, N Engl J Med, 2015;372:2509-20.

20. Reck M, Rodriguez/Abreu D, Robinson AG, et al., Pembrolizumab versus chemotherapy for PD-L1-positive non-small cell lung cancer, N Engl J Med, 2016;375:1823-33.

21. Rosenberg JE, Hoffman-Censits J, Powles T, et al., Atezolizumab in patients with locally advanced and metastatic urothelial carcinoma who have progressed following treatment with platinum-based chemotherapy: a single-arm, multicentre, phase 2 trial, Lancet, 2016:387:1909-20

22. Wolchok JD, Kluger H, Callahan MK, et al., Nivolumab plus ipilimumab in advanced melanoma, N Eng/ J Med, 2013;369:122-33.

23. Postow MA, Chesney J, Pavlick AC, et al., Nivolumab and Ipilimumab versus Ipilimumab in untreated melanoma, N Eng/ J Med, 2015:372:2006-17.

24. D'Angelo SP, Tap WD, Schwartz GK, et al., Sarcoma immunotherapy: past approaches and future directions, Sarcoma, 2014;2914:391967.

25. Lee $\mathrm{S}$, Margolin K, Cytokines in cancer immunotherapy, Cancers, 2011;3:3856-93.

26. Schwinger $\mathrm{W}$, Klass $\mathrm{V}$, Benesch $\mathrm{M}$, et al., Feasibility of high-dose interleukin-2 in heavily pretreated pediatric cancer patients, Ann Oncol, 2005;16:1199-206

27. Ito $\mathrm{H}$, Murakami $\mathrm{K}$, Yanagawa T, et al., Effect of human leukocyte interferon on the metastatic lung tumor of osteosarcoma: case reports, Cancer, 1980:46:1562-5

28. Muller $\mathrm{CR}$, Smeland $\mathrm{S}$, Bauer $\mathrm{HC}$, et al., Interferon- $\alpha$ as the only adjuvant treatment in high-grade osteosarcoma: Iong term results of the Karolinska hospital series, Acta Oncologica, 2005;44:475-80.

29. Bielack SS, Smeland S, Whelan JS, et al., Methotrexate, doxorubicin, and cisplatin (MAP) plus maintenance pegylated interferon alfa-2b versus MAP alone in patients with resectable high-grade osteosarcoma and good histologic response to preoperative MAP: first results of the EURAMOS-1 good response randomized controlled trial, J Clin Oncol, 2015;33:2279-87.

30. Meyers PA, Schwartz CL, Krailo MD, et al., Osteosarcoma: the addition of muramyl tripeptide to chemotherapy improves overall survival - a report from the children's oncology group, I Clin
Oncol, 2008;26:633-8.

31. Allavena P, Signorelli M, Chieppa M, et al., Anti-inflammatory properties of the novel antitumor agent yondelis (trabectedin): inhibition of macrophage differentiation and cytokine production, Cancer Res, 2005;65:2964-71.

32. Suminoe A, Matsuzako A, Hattori $\mathrm{H}$, et al., Immunotherapy with autologous dendritic cells and tumor antigens for children with refractory malignant solid tumors, Pediatr Transplant, 2009;13:746-53.

33. Dillman R, Barth N, Selvan S, et al., A phase I/II trial of autologous tumor cell line-derived vaccines for recurrent or metastatic sarcomas, Cancer Biother Radiopharm, 2004;19:581-8.

34. Robbins PF, Morgan RA, Feldman SA, et al., Tumor regression in patients with metastatic synovial cell sarcoma and melanoma using genetically engineered lymphocytes reactive with NY-ESO-1, J Clin Oncol, 2011;29:917-24.

35. Gatti RA, Good RA, Occurrence of malignancy in immunodeficiency diseases. A Literature Review, Cancer, 1971;28:89-98.

36. Rubinstein PG, Aboulafia DM, Zloza A, Malignancies in HIV/AIDS: from epidemiology to therapeutic challenges, AIDS, 2014;28:453-65.

37. Penn J, Sarcomas in organ allograft recipients, Transplantation, 1995;60:1485-91.

38. Sorbye SW, Kilvaer T, Valkov A, et al., Prognostic impact of lymphocytes in soft tissue sarcomas, PLOS One, 2011;6:e14611

39. Berghuis D, Santos SJ, Baelde HJ, et al., Pro-inflammatory chemokine-chemokine receptor interactions within the Ewing sarcoma microenvironment determine CD8(+) T-lymphocyte infiltration and affect tumour progression, J Pathol, 2011;223:347-57.

40. Tseng WW, Malu S, Zhang M, et al., Analysis of the intratumoral adaptive immune response in well differentiated and dedifferentiated retroperitoneal liposarcoma, Sarcoma 2015:2015:547460.

41. D'Angelo SP, Shoushtari AN, Agaram NP, et al., Prevalence of tumor-infiltrating lymphocytes and PD-L1 expression in the soft tissue sarcoma microenvironment, Hum Pathol, 2015;46:357-65

42. Feng Y, Shen J, Gao Y, et al., Expression of programmed death ligand 1 (PD-L1) and prevalence of tumor-infiltrating lymphocytes (TILS) in chordoma, Oncotarget, 2015;6:11139-49.

43. Fuji $\mathrm{H}$, Arakawa A, Utsumi $\mathrm{D}$, et al., Cd8+ tumor-infiltrating lymphocytes at primary sites as a possible prognostic factor of cutaneous angiosarcoma, Int $J$ Cancer, 2014;134:2393-402.

44. Zitvogel L, Rusakiewicz S, Routy B, et al., Immunological off-target effects of imatinib, Nat Rev Clin Oncol, 2016;13:431-46.

45. Balachandran VP, Cavnar MJ, Zeng S, et al., Imatinib potentiates anti-tumor T cell response in gastrointestinal stromal tumo through the inhibition of IDO, Nat Med, 2011;17:1094-100.

46. Van der Bruggen $\mathrm{P}$, Traversari $\mathrm{C}$, Chomez $\mathrm{P}$, et al., A gene encoding an antigen recognized by cytolytic $T$ lymphocytes on a human melanoma, Science, 1991;254:1643-7.

47. Vigneron $\mathrm{N}$, Human tumor antigens and cancer immunotherapy, Biomed Res Int, 2015;2015:948501.

48. Jacobs JFM, Brasseur F, Hulsbergen-van de Kaa CA, et al., Cancer-germline gene expression in pediatric solid tumors using quantitative real-time PCR, Int I Cancer, 2007;120:67-75.

49. Skubitz KM, Pambuccian S, Carlos JC, et al., Identification of heterogeneity among soft tissue sarcomas by gene expression profiles from different tumors, J Translat Med, 2008:6:23.

50. Maki RG, Soft tissue sarcoma as model disease to examine cancer immunotherapy, Curr Opin Oncol, 2001;13:270-4.

51. Snyder A, Makarov V, Merghoub T, et al., Genetic basis for clinical response to CTLA-4 blockade in melanoma, N Eng/ J Med 2014;371:2189-99

52. Brown SD, Warren RL, Gibb EA, et al., Neo-antigens predicted by tumor genome meta-analysis correlate with increased patient survival, Genome Res, 2014:24:743-50.

53. Rizvi NA, Hellmann MD, Snyder A, et al., Cancer immunology. Mutational landscape determines sensitivity do PD-1 blockade in non-small cell lung cancer, Science, 2015;348:124-8.

54. McGranahan N, Furness AJS, Rosenthal R, et al., Clonal neoantigens elicit $\mathrm{T}$ cell immunoreactivity and sensitivity to immune checkpoint blockade, Science, 2016;351:1463-9.

55. Taylor BS, Barretina J, Maki RG, et al., Advances in sarcoma genomics and new therapeutic targets, Nat Rev Cancer, 2011;11:541-57.

56. Jain $S, X U R$, Prieto VG, Lee P, Molecular classification of soft tissue sarcomas and its clinical applications, Int J Clin Exp Pathol 2010;3:416-28

57. Ohali A, Avigad S, Cohen $I H$, High frequency of genomic instability in Ewing family of tumors, Cancer Genet Cytogenet, 2004;150:50-6.

58. Davoli T, Uno H, Wooten EC, Elledge SJ, Tumor aneuploidy correlates with markers of immune evasion and with reduced response to immunotherapy, Science, 2017;355: pii: eaaf8389.

59. Swann JB, Vesely MD, Silva A, et al., Demonstration of inflammation-induced cancer and cancer immunoediting during primary tumorigenesis, Proc Natl Acad SCi U S A, 2008:105:652-6.

60. Gooden MJM, de Bock GH, Leffers N, et al., The prognostic influence of tumor-infiltrating lymphocytes in cancer: a systematic review with meta-analysis, Br I Cancer, 2011:105:93-103.

61. Tumeh PC, Harview $\mathrm{CL}$, Yearley JH, et al., PD-1 blockade induces responses by inhibiting adaptive immune resistance, Nature, 2014;515:568-71.
62. Fridman WH, Pages F, Sautes-Fridman C, et al., The immune contexture in human tumors: Impact on clinical outcome Nat Rev Cancer, 2012;12:298-306.

63. Daud Al, Wolchok JD, Robert C, et al., Programmed deathligand 1 expression and response to the anti-programmed death 1 antibody pembrolizumab in melanoma, $\mathrm{J}$ Clin Oncol, 2016;34:4102-9.

64. Kim JR, Moon YJ, Kwon KS, et al., Tumor infiltrating PD-1 positive lymphocytes and the expression of PD-L1 predict poor prognosis of soft tissue sarcomas, PLoS One, 2013;8:e82870.

65. Movva S, Wen W, Chen W, et al., Multi-platform profiling of over 2000 sarcomas: identification of biomarkers and novel therapeutic targets, Oncotarget, 2015;6:12234-47.

66. Paydas S, Bagir EK, Deveci MA, et al., Clinical and prognostic significance of PD-1 and PD-L1 expression in sarcomas. Med Oncol, 2016:33:93.

67. Toulmonde M, Adam J, Bessede A, et al., Integrative assessment of expression and prognostic value of PDL1, IDO and kynurenine in 371 primary soft tissue sarcomas with genomic complexity, J Clin Oncol, 2016;34 (suppl; abstr 11008).

68. Dasanu CA, Sustained clinico-radiologic response to anti-cytotoxic $\mathrm{T}$ lymphocyte antigen 4 antibody therapy in metastatic myxoid liposarcoma, J Oncol Pharm Practice, 2016:1-2.

69. McCaughan GJB, Fulham MJ, Mahar A, et al., Programmed cell death-1 blockade in recurrent disseminated Ewing sarcoma, J Hematol Oncol, 2016;9:48-53

70. Miao D, Addegbe D, Rodig SJ, et al., Response and oligoclona resistance to pembrolizumab in uterine leiomyosarcoma: genomic, neoantigen, and immunohistochemical evaluation, J Clin Oncol, 34,2016 (suppl; abstr 11043).

71. Maki RG, Jungbluth AA, Gnjatic $S$, et al., A pilot study of anti-CTLA4 antibody ipilimumab in patients with synovial sarcoma, Sarcoma 2013;2013:168145

72. Merchant MS, Wright M, Baird K, et al., Phase I clinical trial of pilimumab in pediatric patients with advanced solid tumors, Clin Cancer Res, 2016;22:1364-70

73. D'Angelo SP, Shoushtari NA, Keohan ML, et al., Combined KIT and CTLA-4 blockade in patients with refractory GIST and other advanced sarcomas: a phase Ib study of dasatinib plus ipilimumab, Clin Cancer Res, 2016; doi: 10.1158/1078-0432: Epub ahead of print.

74. Paoluzzi L, Cacavio A, Ghesani M, et al., Response to anti-PD1 therapy with nivolumab in metastatic sarcomas, Clin Sarcoma Res, 2016;6:24

75. Tawbi HA, Burgess MA, Corwley J, et al., Safety and efficacy of PD-1 blockade using pembrolizumab in patients with advanced soft tissue (STS) and bone sarcomas (BS): results of SARC028-A multicenter phase II study, J Clin Oncol, 34, 2016 (suppl; abstr 11006)

76. Patnaik A, Kang SP, Rasco D, et al., A phase I study of pembrolizumab (MK-3475); anti-PD-1 monoclonal antibody) in patients with advanced solid tumors, Clin Cancer Res, 2015;21:4286-93.

77. George S, Barysauskas CM, Solomon S, et al., Phase 2 study of nivolumab in metastatic leiomyosarcoma of the uterus, J Clin Oncol 34, 2016 (suppl; abstr 11007)

78. Mellman I, Coukos G, Dranoff G, Cancer immunotherapy comes of age, Nature, 2011;480:480-9.

79. Blankenstein T, Coulie PG, Gilboa E, Jaffee EM, The determinants of tumor immunogenicity, Nat Rev Cancer, 2012;12:307-13.

80. Robbins PF, Morgan RA, Feldman AS, et al., Tumor regression in patients with metastatic synovial cell sarcoma and melanoma using genetically engineered lymphocytes reactive with NY-ESO-1, I Clin Oncol, 2011:29:917-24.

81. Davila ML, Brentjens R, Wang R, et al., How do CARs work? Early insights from recent clinical studies targeting CD19, Oncoimmunology, 2012;1:1577-83.

82. Lettieri CK, Hingorani P, Kolb EA, Progress of oncolytic viruses in sarcomas. Expert Rev, Anticancer Ther, 2012;12:229-42.

83. Nemunaitis J, Tong AW, Nemunaitis M, et al., A phase I study of telomerase-specific replication competent oncolytic adenovirus (telomelysin) for various solid tumors, $\mathrm{Mol}$ Ther 2010;18:429-34

84. Currier MA, Adams LC, Mahller YY, Cripe TP, Widespread intratumoral virus distribution with fractionated injection enables local control of large human rhabdomyosarcoma xenografts by oncolytic herpes simplex viruses, Cancer Gene Ther. 2005;12:407-16

85. Puzanov I, Milhem MM, Minor D, et al., Talimogene laherparepvec in combination with Ipilimumab in previously untreated, unresectable stage IIIB-IV melanoma, J clin Oncol, 2016:34:2619-26.

86. Long GV, Dummer R, Ribas A, et al., Efficacy analysis of MASTERKEY-265 phase $1 \mathrm{~b}$ study of talimogene laherparepvec (T-VEC) and pembrolizumab (pembro) for unresectable stage IIIB-IV melanoma, J Clin Oncol, 2016;34 (suppl; abstr 9568).

87. Krishnadas DK, Bao L, Bai F, et al., Decitabine facilitates imune recognition of sarcoma cells by upregulating CT antigens, MHC moleculaes, and ICAM-1, Tumour Biol, 2014;35:5353-62.

88. Krishnadas DK, Shusterman S, Bai F, et al., A phase I trial combining decitabine/dendritic cell vaccine targeting MAGE-A1, MAGE-A3 and NY-ESO-1 for children with relapsed or therapy-refractory neuroblastoma and sarcoma, Cancer Immuno Immunother, 2015;64:1251-60 